\title{
Recognizing Human Touching Behaviors using a Haptic Interface for a Pet-robot
}

\author{
Futoshi Naya Junji Yamato Kazuhiko Shinozawa \\ NTT Communication Science Laboratories \\ 2-4 Hikaridai, Seika-cho, Soraku-gun, Kyoto, 619-0237, JAPAN \\ E-mail Address: \{naya, junji, shino\}@cslab.kecl.ntt.co.jp
}

\begin{abstract}
This paper presents the preliminary results of classifying human touching behaviors using a haptic interface for a pet-like robot. The haptic interface uses gridded pressure-sensitive conductive ink sheets. Features of the measured pressure data are determined for classification in terms of 1) absolute values, 2) spatial distributions and 3) the temporal differences in measured pressure patterns. Touching behaviors include "slap," "pat," "stroke" and so forth. The experimental results show that a reliable classification of these touching patterns can be accomplished by using the sensor sheet and pressure features.

The results of classification can be used as reward signals for reinforcement learning in controlling the behaviors of a pet-like robot that interacts with humans.
\end{abstract}

\section{INTRODUCTION}

Haptic information is expected to play a great role in enriching communication between humans and robots in the real world. In interactions between humans and robots, distinguishing haptic patterns is very important. In particular, this is useful for helping a robot to recognize human touching behaviors and also for having a robot to express its internal states, or artificial emotions, by physically touching humans. We are currently promoting the Communication Robot project. The goal of this project is to measure human emotions through the physical interaction between humans and robots and also to develop a robot that can naturally interact with humans through touch.

There has been much research on haptic devices designed to develop tactile sensors for robot hands and fingertips $[1,4]$. These sensors have mainly been designed to detect contact between a robot's hands and the target objects to be grasped or manipulated. However, our goal is to have a robot recognize human touch, which means we need to develop tactile sensors that have a relatively wide sensing area and the flexibility to be used to cover the entire body of the robot.

Few sensors have been developed for measuring human touching behavior. Inaba et al.[2] have developed a tactile sensor suit that uses electric conductive fabric to cover the whole body of a humanoid robot. This sensor suit is flexible and has 192 sensing regions that are binary switches. The sensor signals are gathered and superimposed on a visual image of the robot so that the sampling ratio of the sensor data is equal to that of the video frame rate $(30[\mathrm{~Hz}])$. The study presented sensing pattern examples of a human touching the robot's arms, or of the robot holding a ball.

In another study, Shibata et al. [3] proposed a tactile sensor for a pet-like robot. This tactile sensor uses a semiconductor pressure sensor that is connected to a balloon covered by synthetic skin. This sensor is designed to be comfortable for humans. The study presented the sensing patterns of human touching behaviors such as 'patting' and 'stroking', but did not give much detail on the recognition scheme for these behaviors.

In order for a robot to recognize more sensitive patterns of human touch (e.g. a tickle, scratch, etc.), haptic devices are needed to measure touching pressure at a high sampling rate and at high spatial and pressure resolutions.

In this paper, we investigate what is required for haptic devices to recognize human touch. We then show the preliminary results of recognizing typical human touching behaviors including "slap," "stroke" etc. This is accomplished using a gridded pressure sensitive ink sheet. This sensor sheet is thin and flexible for covering the body of a robot, and its resolution and sampling rate is high enough to recognize human touching behaviors. In the following sections, the experimental results are shown for five touching behaviors, and the feature selection used for recognizing these behaviors is discussed.

\section{EXPERIMENTS}

\section{$2.1 \quad$ Experiment setup}

The sensors we used for the following experiments are gridded pressure sensitive ink sheets (NITTA Corp., ISCAN series), as shown in Figure 1. The interface of the sensor is standard ISA or PCI bus of PCs. The size of the sensor area of the sheet is $16.5 \times 16.5 \mathrm{~cm}^{2}$ and about $0.3 \mathrm{~mm}$ thick. The sensor area is gridded in $44 \mathrm{x}$ $44=1936$ elements, so one gridded element size is 3.75 $\mathrm{x} 3.75 \mathrm{~mm}^{2}$. The measuring pressure range is $0.2-2.0$ $\left[\mathrm{kgf} / \mathrm{cm}^{2}\right]$. The pressure values can be retrieved at a rate of $127[\mathrm{~Hz}]$ (maximum), and the output is given as 
a distribution as raw data of 256 scaled values. The size and the measuring range of this sensor are suitable for measuring the touching behaviors of human hands. In the following sections, we denote $F_{i j}(t)$ as the spatial pressure pattern of the frame at time $t$.

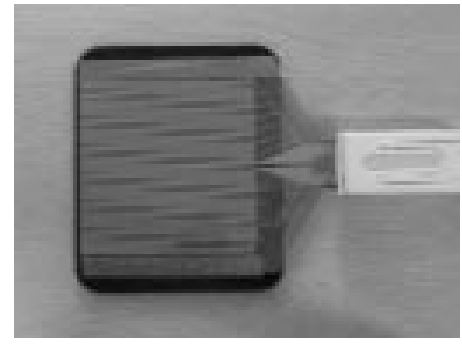

Figure 1: Sensor sheet (NITTA Corp., I-SCAN)

\subsection{Touching behaviors and experi- ment}

We measured five different touching behaviors for 11 subjects (six males and five females). The touch behaviors are "slap," "pat," "scratch," "stroke" and "tickle". Each subject was told to repeat each behavior to the sensor in five seconds. The pressure data was then sampled at a rate of $120[\mathrm{~Hz}]$. The number of the total frame sequence was $600(120 \times 5)$. A typical 'slapping' pattern sequence is shown in Figure 2. This slapping sequence includes 18 frames $(=150[\mathrm{msec}])$. As shown in this figure, the sampling rate is fast enough to capture steep pressure changes in slapping.
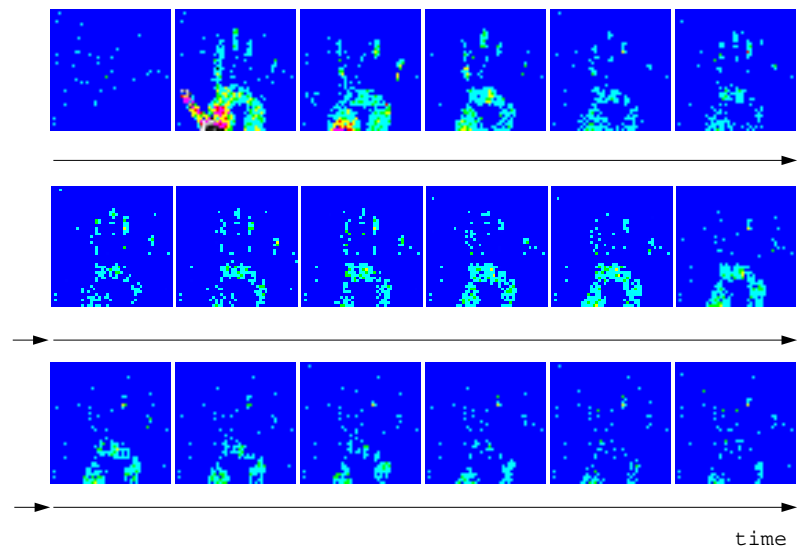

Figure 2: A typical "slap" sequence.

$(1$ frame $=1 / 120[\mathrm{sec}] \simeq 8.3[\mathrm{msec}])$

\section{$2.3 \quad$ Feature selection}

In order to recognize human touching behaviors in realtime, we need to determine pressure features that can be processed by non-iterative and non-time-consuming processes. As the first standpoint for determining feature space, we use the following values as the basic features of a pressure distribution:

1. $w(t)$ : The total load of a frame at time $t$

$$
w(t)=\sum_{i} \sum_{j} F_{i j}(t) \quad(1 \leq i, j \leq 44)
$$

2. $a(t)$ : The total contact area of a frame at time $t$

$$
\begin{gathered}
a(t)=\sum_{i} \sum_{j} b_{i j}(t) \quad(1 \leq i, j \leq 44) \quad \text { where } \\
b_{i j}(t)= \begin{cases}1, & \text { for } F_{i j}(t) \geq f_{\text {thresh }} \\
0, & \text { otherwise. }\end{cases}
\end{gathered}
$$

Here, $b_{i j}(t)$ corresponds to the binary image of the pressure distribution at time $t$. To reduce noise in the pressure distribution, the threshold value of $f_{\text {thresh }}$ is determined from measuring $F_{i j}$ when no weight is loaded onto the sensor.

Figure 3 and 4 show sample touching sequences by a male and a female, respectively. The left column of these figures shows the total load changes $w(t)$ of each of the five behaviors, and the right column shows the changes of the total contact area $a(t)$ for the corresponding behaviors. Each sequence of the figure contains several touching actions, each of which represents changes in the pressure data caused by one behavior action.

As expected, there are many varieties of pressure patterns for the same behavior. Behaviors such as "slap" have common features such as steep changes in pressure values (Figure $3(\mathrm{a})(\mathrm{b})$ and Figure $4(\mathrm{a})(\mathrm{b}))$. On the other hand, behaviors like "stroke" and "scratch" differ from person to person. For example, the behavior may be alternately repeated while keeping touch with the sensor (Figure $3(\mathrm{~d})$ ) or it may be a one-directional touch-and-release motion (Figure $4(\mathrm{~d}))$.

From Figure 3 and 4, behaviors such as "slap" and "pat", and some cases of "scratch" and "stroke" demonstrate tendencies to have temporal pressure patterns specific to each behavior. However, we still need to manage the following characteristics, which differ even among pressure data from the same behavior:

1. Intensity of each touching pattern

2. Duration and frequency of each touching pattern

3. Motion direction and motion range of each touching pattern 

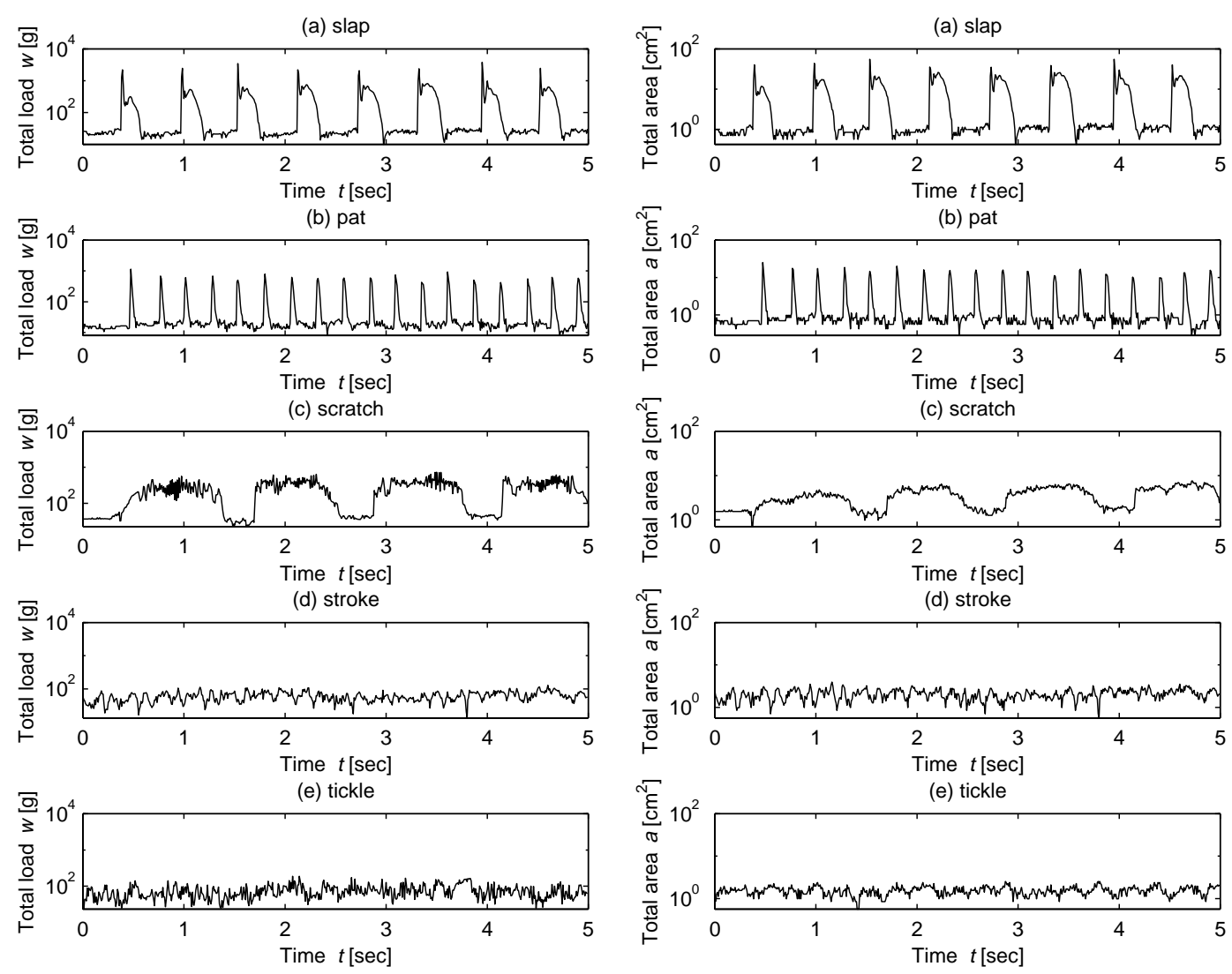

Figure 3: Sample touching patterns by a male.
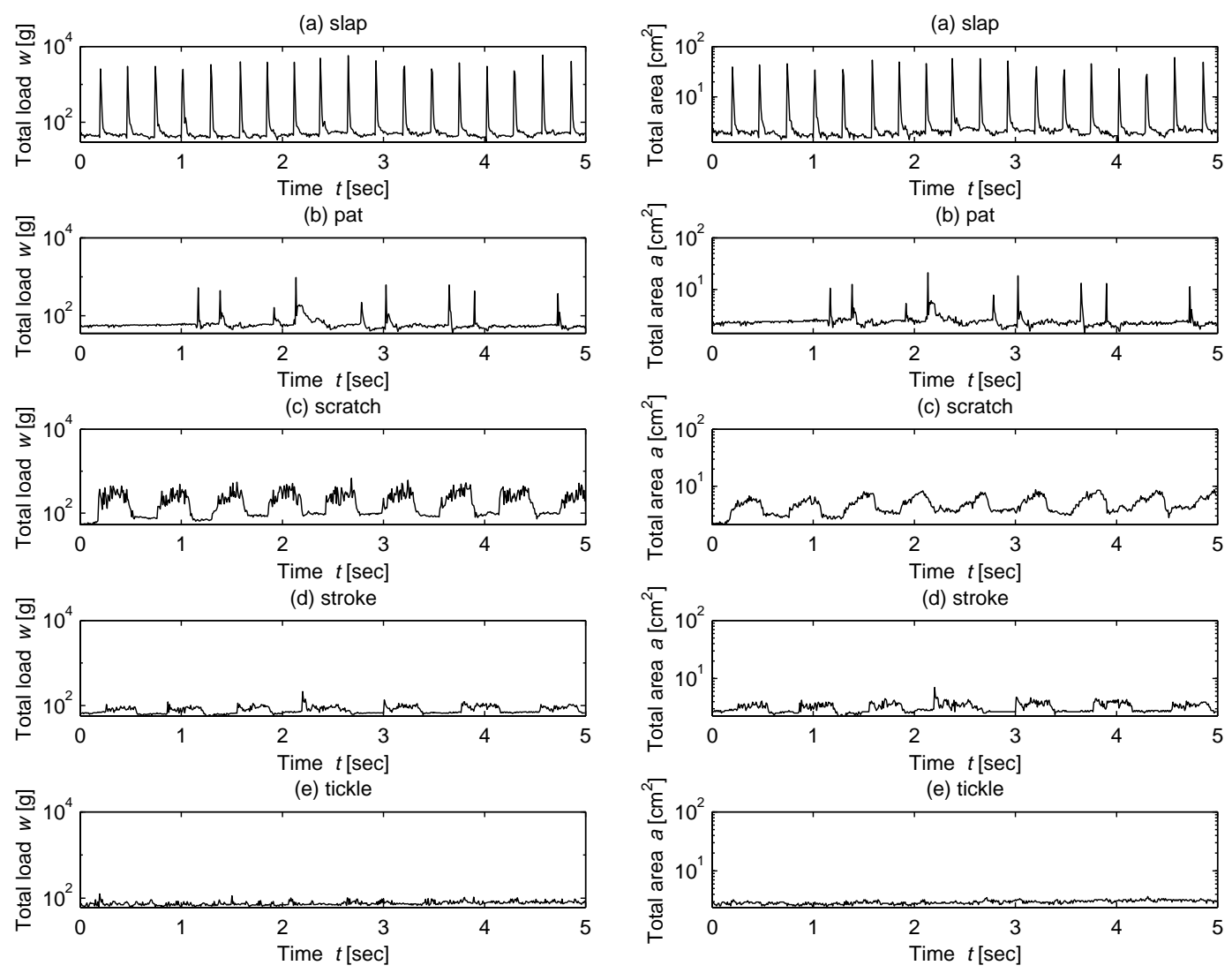

Figure 4: Sample touching patterns by a female. 
In order to cope with these differences, a recognition process needs to have features that are independent of these characteristics. Here, we focus on the steepness of each touching pattern in terms of absolute values and the temporal changes in the pressure data measured.

\section{CLASSIFYING HUMAN TOUCHING BEHAVIORS}

\subsection{Classification results using absolute pressure values}

As a feature relating to steepness, we first sampled the peak values of the total load $w_{\text {peak }}(t)$ and the corresponding values of the total area $a_{\text {peak }}(t)$. The temporal duration for finding peaks is determined to be 30 frames $(250[\mathrm{msec}])$ after the total load of a frame exceeds the threshold value $(50[\mathrm{~g}])$.

For each subject, these feature values were estimated by averaging several touching actions of each behavior. The results are shown in Figure 5.

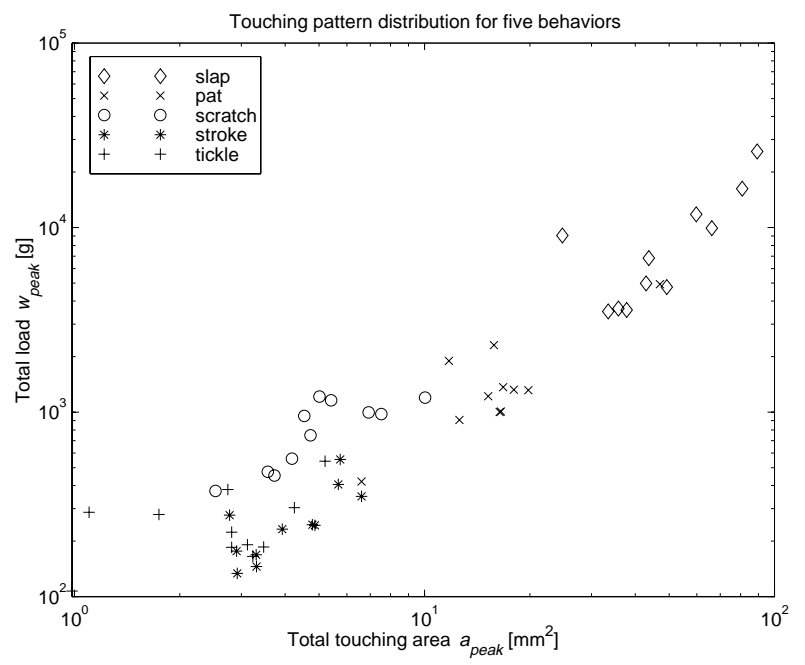

Figure 5: Touching pattern distribution. (Total area vs. Total load)

In Figure 5, each point represents the average value of the $a_{\text {peak }}$ and $w_{\text {peak }}$ of each behavior for one subject. Table 1 shows the mean value and standard deviation of the total area and the total load for each behavior among the 11 subjects. Although the data points are somewhat scattered, the data points of "slap," "pat" and "scratch" seem to be linearly separated.

For classifying these sampled data, we used $k$ nearest neighbor $(k$-NN) method with leaving-one-out. The recognition ratio for each behavior is shown in Table 2 .

As it is obvious from Figure 5 and Table 2, the peaks of the total load and area are not good features

\begin{tabular}{|c||r|r|r|r|}
\hline Behavior & $\bar{w}[g]$ & $\sigma_{w}[\mathrm{~g}]$ & $\bar{a}\left[\mathrm{~mm}^{2}\right]$ & $\sigma_{a}\left[\mathrm{~mm}^{2}\right]$ \\
\hline \hline Slap & 9112.3 & 6879.0 & 51.3 & 20.4 \\
\hline Pat & 1608.2 & 1210.9 & 17.9 & 10.3 \\
\hline Scratch & 828.0 & 318.1 & 5.3 & 2.0 \\
\hline Stroke & 266.5 & 127.0 & 4.3 & 1.3 \\
\hline Tickle & 259.0 & 121.0 & 2.9 & 1.3 \\
\hline
\end{tabular}

Table 1: Mean and standard deviation of the peak values of the total load and total area of each behavior.

\begin{tabular}{|c||r|r|r|r|r|}
\hline Behaviors & Slap & Tap & Scratch & Stroke & Tickle \\
\hline \hline $\begin{array}{c}\text { Recog. } \\
\text { ratio(\%) }\end{array}$ & 100.0 & 63.6 & 72.7 & 36.4 & 27.3 \\
\hline
\end{tabular}

Table 2: Recognition ratio of the classifying behaviors based on the data.

for discriminating between "stroke" and "tickle" behaviors. As we can see from Figure 3 and 5, the absolute values of the total pressure and area of these two behaviors are very similar among all the subjects. This is because peaks do not contain the temporal information of the movement of the pressure data. Thus, we need to introduce another feature vector to distinguish between "stroke" and "tickle".

\subsection{Classification results using tempo- ral differences in pressure values}

It is possible to use some motion-related features such as the centroid of pressure images, however, such features are not so stable through a sequence of behaviors in which fingertips touch on and off, as in stroking or tickling.

To introduce the temporal information in the pressure patterns, we used the difference between peaks of the total load $w_{\text {peak }}$ and the total area $a_{\text {peak }}$ at time $t$ and $t-1$.

We define the normalized temporal difference of the total load and the total area at the peak points as follows:

- $\delta w_{\text {peak }}(t)$ : normalized temporal difference of the total load between time $t$ and $t-1$.

$$
\delta w_{\text {peak }}(t)=\frac{\sum_{i j}\left|F_{i j}(t)-F_{i j}(t-1)\right|}{\sum_{i j} F_{i j}(t)},
$$

where $t$ gives a peak value of $\sum_{i j} F_{i j}(t)$.

- $\delta a_{\text {peak }}(t)$ : normalized temporal difference of the total area between time $t$ and $t-1$.

$$
\delta a_{\text {peak }}(t)=\frac{\sum_{i j}\left|b_{i j}(t)-b_{i j}(t-1)\right|}{\sum_{i j} b_{i j}(t)},
$$

where $t$ is the same as that above and has a peak at $\sum_{i j} F_{i j}(t)$. 
Figure 6 shows the distribution of the "stroke" and "tickle" touching patterns for 11 subjects by using these features. By applying Fisher's linear discriminant method to these data, these two behaviors can be clearly separated by using these temporal difference features. Thus, by combining two discriminant methods above, the averaged recognition ratio of five touching behaviors is $87.3 \%$.

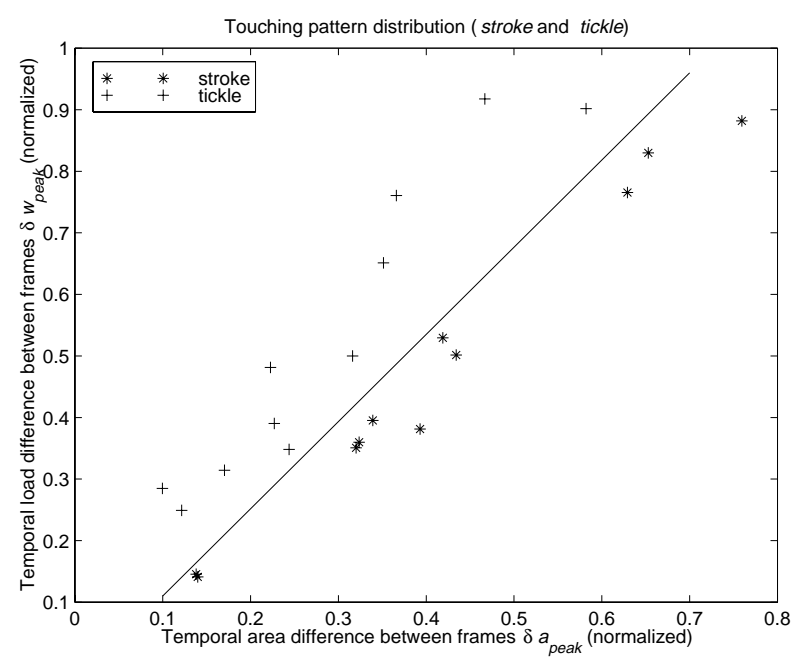

Figure 6: Touching pattern distribution. Temporal difference of the total area (normalized) vs. temporal difference of the total load (normalized).

\section{CONCLUDING REMARKS AND FUTURE WORK}

In this paper, five different human touching behaviors were measured for 11 subjects by using gridded pressure-sensitive conductive ink sheets. The classification results of these behaviors are presented by defining the features of the absolute values and the temporal differences in the pressure distribution of the sensor.

For actions like "slap," "pat" and "scratch," it was found that it is possible to discriminate between these behaviors by using contact area and absolute pressure values as gross features. To classify more sensitive touching behaviors such as "stroke" and "tickle," temporal differences in the pressure patterns and contact area need to be selected as features. These features are very simple to extract and are not computer intensive, so realtime recognition among these behaviors is possible using these features.

We are currently developing "communication robots" and are focusing on the functions that are necessary for a robot to communicate with humans through the physical interaction (Figure 7). In future work, we plan to include to equip the whole robot body with sensor sheets and to measure human emotions from the recognition results of human touching behav- iors. We are also planning to use these recognition results as reward signals for controlling the behavior of a robot.

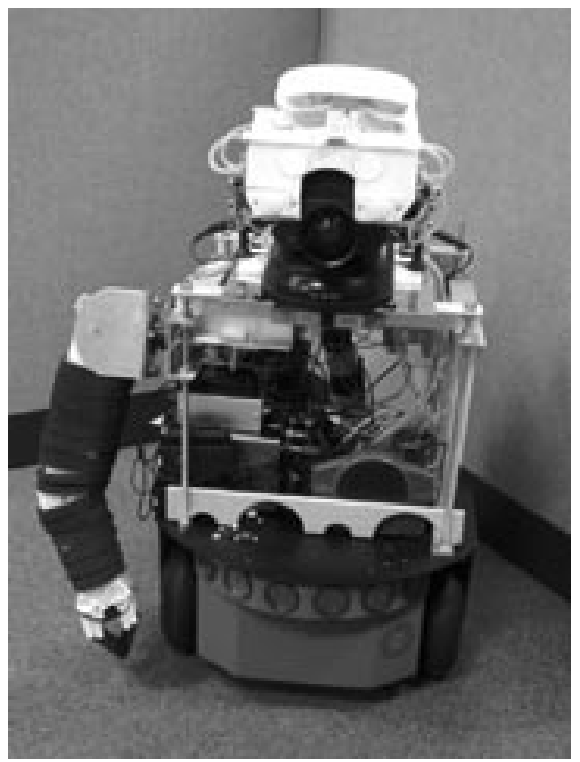

Figure 7: Communication Robot (Kenzo)

\section{REFERENCES}

[1] E. Cheung and V. Lumelsky, "Development of sensitive skin for a 3D robot arm operating in an uncertain environment," Proceedings of the IEEE International Conference on Robots and Automation, (ICRA'89), pp. 1056-1061, 1989.

[2] M. Inaba, Y. Hoshino, K. Nagasaka, T. Ninomiya, S. Kagami, and H. Inoue, "A Full-Body Tactile Sensor Suit Using Electrically Conductive Fabric and Strings," Proceedings of the IEEE/RSJ International Conference on Intelligent Robots and Systems, (IROS'96), pp. 450457, 1996.

[3] T. Shibata, M. Yoshida, and J. Yamato, "Artificial Emotional Creature for Human-Machine Interaction," Proceedings of the IEEE International Conference on Systems, Man, and Cybernetics (SMC'97), pp. 22692274, 1997.

[4] J. Son, E. A. Monteverde, and R. D. Howe, " A tactile sensor for localizing transient events in manipulation", Proceedings of the 1994 IEEE International Conference on Robotics and Automation (ICRA'94), pp. 471-476, 1994. 